\title{
Dimension of time in strange attractors
}

\author{
Robert J. Krawczyk \\ College of Architecture, Illinois Institute of Technology, Chicago, USA \\ Correspondence to: Robert J. Krawczyk. College of Architecture, Illinois Institute of Technology, Chicago, USA. Email: bitartworks@gmail.com.
}

Submitted Mar 13, 2019. Accepted for publication Mar 21, 2019.

doi: $10.21037 /$ cdt.2019.03.03

View this article at: http://dx.doi.org/10.21037/cdt.2019.03.03

Scientific phenomenon has an artistic aesthetic that transcends its ability to attempt to explain the world around us.

Color records time and creates the illusion of dimensionally.

Science is always searching for predictability. For example, when will the next solar eclipse occur? Science can predict the exact time and also tell us where on earth the best place to view it is. Scientific theory relies on whether predictions are supported by observation. These theories rely on relationships that are deterministic; given a present condition, a future event can be determined.

Then we come to what has been dubbed the butterfly effect. Is it possible for a butterfly flapping its wings in Brazil to set off tornadoes in Texas?

Chaotic processes are not random; they follow rules, but even very simple rules can produce extreme complexity. This complexity can be expressed as a series of equations or visualized and rendered when the element of time as color is introduced into its interpretation. The mathematics of chaos provides the tools for creating and displaying such phenomenon.

Time becomes color.

Strange attractors generate repeating point patterns in two-dimensional space while their coloring algorithms which represent time can produce images of coherent threedimensional forms. The third dimension is determined by the perception of the viewer coupled with a created intent. These forms can include a ghostly view into an imaginary core. The swirling patterns gently display possible subsurface structures that cannot be logically followed through any dimension. Dimensions become ambiguous as viewer's perception attempts to combine the individual points so they complete a whole, one that is devoid of context.

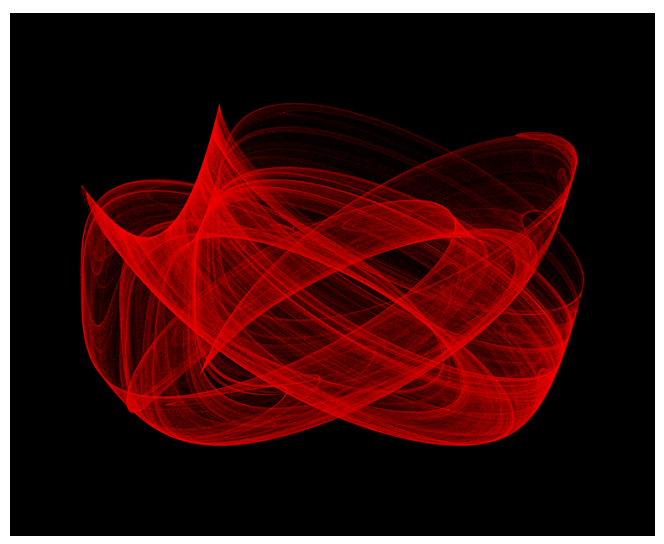

Figure 1 Bends Through III.

Many of these images could have been inspired from natural forces such as, wind and water, or earthen formations (Figures 1-5). For example, the Stone Series explores the possible subsurface patterns in nature that are not visible to the human eye; the Smoldering and Gathering images smoke, others; folding, bending, twisting, draping and crumpling of identifiable materials or organisms.

These images were created algorithmically using a series of related strange attractor equations with coloring schemes that bring out the time element of the computational process. Each was developed by the computation of between 12 to 57 million points. A total of 24,000 images were generated from 24 equations. Each image was reviewed and then approximately 200 were selected for final rendering.

Robert is a Professor at the College of Architecture at the Illinois Institute of Technology in Chicago focusing on digital craftmanship. During his 35 years at IIT, he has developed and taught a series of computer-aided design and digital design courses. His research into digital methods in the disciplines of science, mathematics, architecture, 


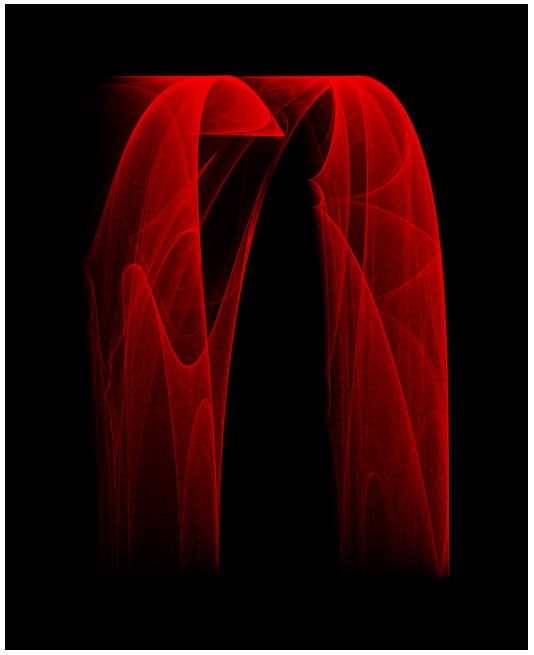

Figure 2 Draping Fall II.

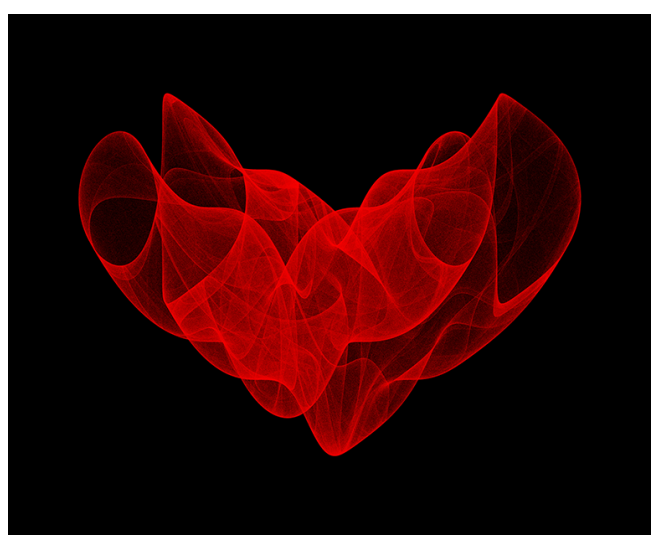

Figure 3 Gathering Rise I.

art, and technology has been published and presented internationally in the form of prints, web pieces, sculptural, and architectural studies. He has been an exhibiting digital artist since 1997 .

His artwork can be found at: www.BitArtWorks.com and prints from this series are available at: robert-krawczyk. pixels.com and can be contacted at: bitartworks@gmail.com.

\section{Acknowledgments}

None.

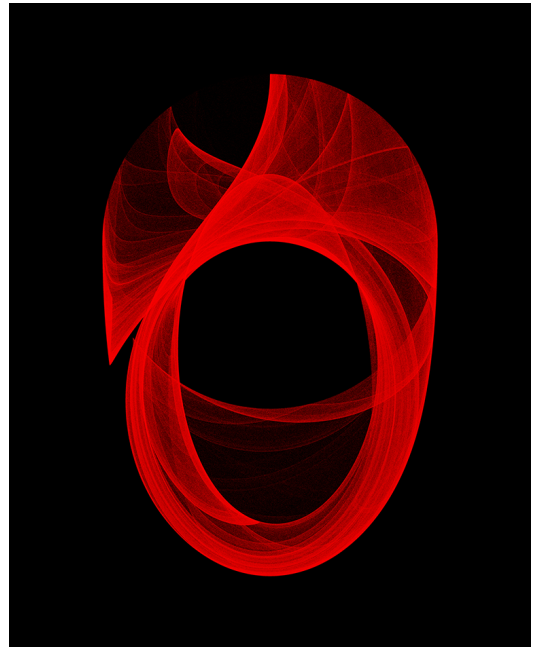

Figure 4 Ring Unraveling II.

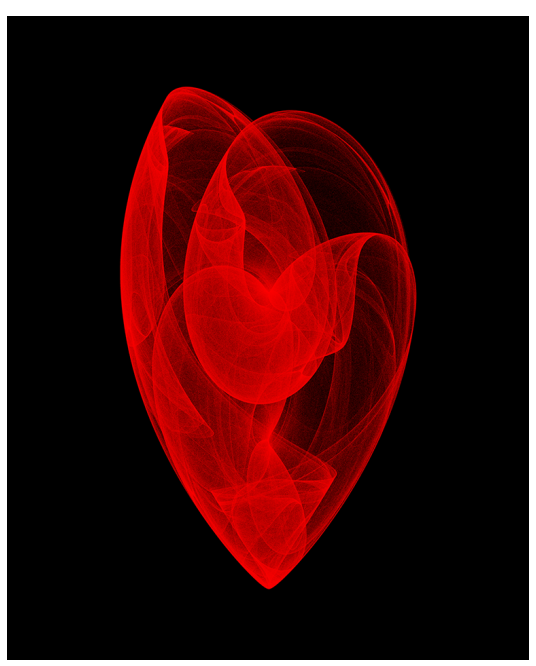

Figure 5 Within Shell II.

\section{Footnote}

Conflicts of Interest: The author has no conflicts of interest to declare.

Cite this article as: Krawczyk RJ. Dimension of time in strange attractors. Cardiovasc Diagn Ther 2019;9(5):533-534. doi: $10.21037 / \mathrm{cdt} .2019 .03 .03$ 